\title{
La «crisis» del siglo III en la historiografía española
}

\author{
Yolanda Peña Cervantes *
}

\begin{abstract}
RESUMEN
ABSTRACT

La consideración del s. III d.C. como

The consideration of the 1 Ird $^{\text {c }}$ century un periodo de profunda crisis ha sido as a period in a deep crisis, has been una constante en la historiografia a permanent topic in the Spanish española hasta bien entrada la década Historiography until the late 1970s. de los setenta. Los motivos en los que se ha asentado esta afirmación y su evolución a lo largo del tiempo, hasta The foundations of this statement and its development along time, until its actual definition or negation su matización o negación en los momentos actuales, constituyen el nowadays, establish the aim of this research.
\end{abstract}

\section{CONSIDERACIONES GENERALES}

El propósito del presente trabajo es realizar un análisis del tratamiento que en la historiografía española recibe la llamada "crisis" del s. III. En principio, nuestra atención se centra en los aspectos vinculados al mundo urbano. Sin embargo, la construcción historiográfica, que se ha mantenido vigente en España hasta bien entrada la década de los setenta, nos obliga a analizar en conjunto la evolución del concepto de "crisis" referida al s. III, ya que ésta se presenta extensible a todos y cada uno de los elementos definidores de la Cultura Clásica, entre los que inevitablemente se encuentra la ciudad.

* Universidad Autónoma de Madrid. 
Aunque, de forma general, se asocie la totalidad del s. III con un periodo de crisis, ésta se circunscribe más exactamente, en la historiografía tradicional, al periodo conocido como Anarquía Militar comprendido entre la muerte de Alejandro Severo (235 d. C) y la subida al trono de Diocleciano (284 d. C.). Durante estos 50 años se suceden los emperadores en Roma, siendo habituales las usurpaciones y los levantamientos, generando un clima de profunda inestabilidad política, a la par que se producen constantes incursiones de pueblos germanos en las fronteras del Imperio. Ésta es la visión convulsa que presentan las fuentes escritas y que, como más tarde veremos, la historiografía ilustrada hará extensiva a todos los ámbitos de la cultura romana.

El s. III se considera un periodo de inflexión en el que nacen las nuevas variables socioculturales y políticas que van a caracterizar al llamado «bajo Imperio". Es, por lo tanto, un periodo clave, ya que el modelo de interpretación histórica que se utilice para su análisis determinará la imagen del Imperio que se proyectará a los siglos posteriores. Esta última reflexión puede ser utilizada también en sentido inverso, ya que el apriorismo histórico ha llevado a analizar este siglo a partir de elementos culturales detectados en el bajo Imperio convirtiéndose, en muchos casos, tan sólo en una mera exégesis del periodo posterior y olvidando su propia idiosincrasia histórica. Ésta es sin lugar a dudas una de las consideraciones que más importancia ha tenido en los estudios dedicados a este periodo. Su carácter de puente entre dos realidades culturales distintas dificulta enormemente la realización de un análisis histórico específico que recoja su riqueza y complejidad.

A pesar de esto, la "crisis" del s. III, a la que tradicionalmente pone fin el gobierno de Diocleciano, se incluye aún en el periodo altoimperial. Todos sabemos ya los inconvenientes y problemas que supone la delimitación artificial del tiempo histórico, y, a la vez, la necesidad metodológica y expositiva que el propio estudio histórico tiene de estos items temporales, siempre y cuando no supongan una división férrea que intervenga en el análisis de los acontecimientos históricos. En este caso, la presentación en obras de síntesis y autoría conjunta del periodo denominado Anarquia Militar como apéndice de la etapa "gloriosa" significada por el alto Imperio y analizada por investigadores no especializados en este periodo tan complejo, ha concluido la fijación de tópicos difícilmente modificables en el imaginario histórico común.

Otro de los problemas que presenta el estudio de este periodo es la falta de fuentes escritas. Contamos con pocos testimonios y, en todos los casos, se trata de noticias que inciden en la idea de inestabilidad y con- 
vulsión política. Desde un punto de vista "historicista" un periodo marcado por un caos político, de la envergadura que nos presentan las fuentes, necesariamente se traduce en una crisis global de todo el sistema. La falta de otro tipo de información histórica que pueda añadir nuevos datos a esta visión se suple acentuando el carácter catastrofista que desarrollan los acontecimientos políticos.

Para el caso de la Península Ibérica, esta carencia de fuentes escritas es aún más aguda. Junto a la ausencia de textos redactados en el mismo s. III, común a todo el Imperio, en nuestro caso la situación periférica de Hispania, alejada de los grandes escenarios de la lucha política y militar, provoca una mayor escasez de noticias que, durante mucho tiempo, ha asfixiado a la historiografía española de este periodo. Tan sólo poseemos vagas menciones que refieren el paso por la Península de pueblos bárbaros, sin que se pueda calibrar el verdadero alcance de estas incursiones a la luz de los textos disponibles.

De esta manera, ni Dion Casio ni Herodiano, los únicos historiadores que escriben en el S. III, aportan datos significativos sobre la "crisis", al fecharse sus relatos en el primer cuarto de siglo. El resto de las fuentes datan de un momento posterior a los hechos narrados. Así, el Libro de los Césares de Aurelio Victor, el Breviario de Eutropio, la Crónica de Jerónimo y la Historia Augusta, todos ellos de la segunda mitad del s. IV, y ya en el s. $v$ la Historia de Orosio y la Crónica de Prospero. Nos encontramos, por tanto, ante una primera dificultad al tratarse de noticias escritas como mínimo un siglo después de producirse los acontecimientos y ya destiladas por la evolución posterior del Imperio. En cualquier caso, junto a una visión catastrofista de los acontecimientos que sacuden al imperio a lo largo del s. III, especialmente tras la muerte de Alejandro Severo, no encontramos referencias sobre la Península Ibérica, si no es para indicar la entrada de pueblos bárbaros que provocan la destrucción de Tarraco. Estas incursiones se producen en todos los casos en el reinado de Galieno y son protagonizadas por francos o germanos según la fuente que consultemos.

Esta escasez de fuentes se combina con un retraso en el conocimiento arqueológico de este periodo. Especialmente relevante es la falta de seriación e identificación de la cerámica producida en la Península durante el siglo III, un problema que aún en nuestros días dista mucho de estar resuelto. Esto ha provocado, que a falta de cerámicas de importación o de materiales numismáticos, muchos estratos se hallan fechado equivocadamente $y$, lo que es más preocupante, que todavía no poseamos la clave para hacerlo correctamente. Hasta 1961 no se pública una primera sínte- 
sis especifica sobre la TSH (Mezquiriz, 1961) lo que supone un considerable atraso respecto de la investigación sobre la sigillata itálica o la gálica. Las producciones de sigillata asociadas al siglo III presentan aún en la actualidad una multitud de problemas derivados, nuevamente, del carácter tradicional de puente de este siglo, esta vez entre las producciones de TSH y TSHT. Si, como señalábamos, el estudio de la sigillata hispánica altoimperial presentaba ya un retraso considerable respecto de otras producciones imperiales, este hecho se agudiza aún más en el caso de las producciones tardias, teniendo que esperar hasta la década de los ochenta para encontrar los primeros estudios que dotan a esta cerámica de una naturaleza específica (Mayet, 1984; López Rodríguez, 1985...) y que permiten afirmar que el siglo III mantiene la producción de TSH. A pesar de esta afirmación, ya arraigada en los últimos estudios (Paz Peralta, 1991; Orfila, 1993; Tuset y Buxeda, 1995; Juan Tobar, 1997...), el problema dista mucho de estar resuelto aunque se hallan detectado algunas de la característica técnicas, que no tipológicas, que definen a la TSH del siglo III. Todavía es imposible ajustar la cronología de estas cerámicas dentro de este marco temporal, para intentar conseguir una secuencia interna, lo que provoca unas dataciones de los fenómenos arqueológicos excesivamente holgadas.

La crisis urbana, tópico en la investigación durante muchos años, se deducía, a partir de un planteamiento positivista, del ambiente general de colapso político y económico expuesto en las fuentes escritas. La falta de excavaciones urbanas, incentivadas a partir de la Ley de Patrimonio de 1985, no era un obstáculo en la mayor parte de la historiografía española para generalizar destrucciones y abandonos que casaran bien con la argumentación histórica de unas invasiones que convulsionaron los cimientos del hasta este momento intocable Imperio Romano. El sentir decadentista en la interpretación del bajo Imperio así lo requería. La investigación arqueológica en nuestro país, vinculada tradicionalmente a corrientes esteticistas y objetuales, ha acumulado un considerable atraso en el estudio del bajo Imperio derivado nuevamente de esta noción de decadencia. Durante muchos años tan sólo algunos elementos específicos de la cultura material de este periodo, como sarcófagos o mosaicos, requirieron un estudio serio por parte de los arqueólogos.

Hemos esbozado hasta aquí algunos elementos característicos del s. III que han determinado la evolución de su estudio histórico-arqueológico. Tanto su indefinición tempo-cultural, como la escasez de fuentes escritas y la incidencia exclusiva que éstas hacen en el caos político que acompaña al Imperio entre los años 235 y 284 , como el retraso acumulado en el estudio de la cultura material condicionan la investigación, independientemente del 
punto de vista desde el que se aborde su estudio. En el fondo, la existencia de una profunda crisis en el s. III sirve como justificante de la decadencia que supone el bajo Imperio. Como ya advertíamos, el apriorismo histórico consigue imponerse en el estudio de este oscuro periodo. Es más, cuando se produzca la superación del mito decadentista del bajo Imperio, ésta vendrá unida necesariamente a la revisión del análisis realizado sobre el s. III, matizando o negando la idea de crisis. Como veremos, la parquedad informativa de este periodo, unida a la excepcional trascendencia que posee para la comprensión del posterior desarrollo del Imperio, hacen que historiográficamente no pueda realizarse un análisis separado de los mismos.

En cuanto a la reflexión historiográfica, la llustración, momento en que surge el concepto de crisis, supondrá un cambio radical en los planteamientos metodológicos y teóricos de la Historia. El empirismo, el positivismo, se instalará a partir de este momento como corriente epistemológica que marcará las directrices de las ciencias sociales, que pretenderán revestirse paulatinamente con los atributos de las llamadas ciencias de la naturaleza. La superación de estos postulados a través de la Hermenéutica y de la Escuela de Frankfurt abrirá un debate que en la actualidad continua vigente y que en el campo de la arqueología adquiere una mayor intensidad debido a los fundamentos metodológicos que la sustentan y a su campo de estudio. No podemos entrar aquí a realizar un análisis pormenorizado de las implicaciones de esta controversia teórica ya que nos apartariamos ostensiblemente del tema objeto de nuestro análisis (Habermas, 1968; Trigger, 1992; Hooder, 1988).

Es necesario, sin embargo, recordar que la validez de cualquier análisis historiográfico pasa por entender que una hipótesis histórica debe interpretarse desde la síntesis entre la producción cultural objeto de estudio y la realidad personal y sociocultural del investigador. Sólo de esta manera la Historiografía adquiere su sentido, no como una mera exposición de los antecedentes históricos para el estudio de un determinado tema sino como explicación de la evolución de la disciplina histórica, de acuerdo al propio contexto en el que se inscribe. En la introducción del II Congreso de Historiografía Española, Ricardo Olmos (Olmos, 1997) realiza unas interesantísimas apreciaciones sobre la necesidad de llevar a cabo una reflexión del sentido de la Historiografía en el panorama actual de la investigación arqueológica. Sin lugar a dudas, las reflexiones de este autor y sus propuestas deben ser el camino a seguir para lograr una disciplina viva y fructífera, que permita realizar una autocrítica positiva desde dentro de la arqueología. Porque de todos es sabido que la salud de una disciplina depende de su capacidad de análisis y revisión de sus principios metodológicos y postulados teóricos. En la actualidad, la Historiografía española 
presenta un inusitado vigor que comienza ya a dar sus frutos. En este sentido conviene destacar los sendos congresos de Historiografía ya realizados, y la creación de la Sociedad Española de Historia de la Arqueología que publica la primera revista monográfica a este respecto (Arcaia).

\section{LA VIGENCIA DE LA CRISIS EN LA HISTORIOGRAFIA ESPAÑOLA}

\section{A) Nacimiento e implantación del concepto de "crisis"}

La idea de una decadencia asociable a una profunda crisis de la cultura clásica nace, sin lugar a dudas, como fruto del profundo sentimiento antireligioso que acompaña a la llustración y de la valoración como elemento absoluto de la razón y su vinculación mítica con el periodo clásico. La lucha abierta entre llustración y Antiguo Régimen traerá consigo una reelaboración de la Historia que se amolda a los nuevos postulados racionalistas. De esta manera Le Beau acuña el termino «Bajo Imperio» en su obra Histoire du Bas-Empire en commençant à Constantin le Grand, redactada entre 1752 y 1817 , con toda la carga peyorativa que esto supone como diferenciación respecto al alto Imperio o etapa gloriosa de la cultura romana. El hecho de que el término haya hecho fortuna, manteniéndose hasta nuestros dias, aunque este despojado ya, en principio, de toda consideración peyorativa, no ejemplifica sino la asunción plena en la historiografía moderna de una noción de decadencia vinculable a los últimos siglos del Imperio y coincidente con la cristianización del mismo.

Pero sin lugar a dudas, es la monumental obra de Gibbon, La Historia del declive y caída del Imperio Romano, la que supone todo un hito historiográfico, no ya sólo por la calidad y la erudición de la misma, sino por su carácter de referente para los estudios posteriores dedicados a este periodo. Frente a la "edad de oro» encarnada por la dinastía de los Antoninos se inicia un largo periodo de decadencia, que él concluye con la toma de Costantinopla en Oriente, y que supone la eliminación paulatina de la cultura romana (Collingwood, 1952). Desde luego no era la primera vez que una obra se dedicaba exhaustivamente a plantear los siglos III, IV y $\vee$ como etapas inmersas en un proceso histórico decadentista. De hecho, en el s. XVI, el historiador renacentista Löwenklav en Apologie pour la défense de Zosime plantea ya la crisis del Imperio Romano con unos postulados muy similares a los utilizados por Gibbon. El primero, tal y como señala Mazzarino (Mazzarino, 1973, 93), ha pasado prácticamente al olvido, mientras que Gibbon constituye aún hoy un punto de referencia ineludibie 
para los estudios del bajo Imperio. Como afirma el propio estudioso italiano, el motivo es que la línea de pensamiento que ambos esgrimen no podía dar sus frutos bajo la mentalidad renacentista, fascinada por la grandeza del mundo clásico pero imbuida aún en un profundo sentir religioso.

La consideración del bajo Imperio (y utilizaremos de ahora en adelante este término por motivos de comodidad y asumiéndolo ya plenamente como una convención histórica descargada de cualquier aspecto peyorativo) como un periodo de crisis nace, o mejor dicho consigue imponerse, en un momento en el que el absolutismo monárquico ha entrado en crisis de la mano de la llustración y la Revolución Francesa. Se produce una conexión obvia por parte de los pensadores (ver por ejemplo Consideraciones sobre las causas de la grandeza de los Romanos y su decadencia de Montesquieu) y los historiadores ilustrados entre sus ideales de gobierno y aquellos a los que pone fin la monarquía de derecho divino encarnada por Constantino. Es evidente, pues, que la institucionalización del Cristianismo por parte del gobierno de Roma, así como la entrada de los pueblos bárbaros, se aprecia como el inicio de un largo periodo de oscurantismo que genera todos aquellos "vicios" del Antiguo Régimen que se están intentando combatir.

Bajo la óptica del pensamiento ilustrado esta conexión entre bajo Imperio y decadencia parece justificada, más difícil es intentar adivinar los motivos que hacen que esta concepción se mantenga vigente en la historiografía europea como mínimo hasta finales de la década de los cincuenta del siglo $\mathrm{XX}$, consiguiendo sobrevivir a profundas renovaciones ideológicas. Ni siquiera el Romanticismo, con su defensa de los valores irracionales y de la Edad Media, palia esta visión de crisis y decadencia. Historiadores pertenecientes a corrientes de pensamiento tan distintas como Burckhardt, Seek, Rostovzeff o Spengler presentan diferentes causas pero una misma argumentación negativa en la valoración de este periodo. Desconocemos qué subyace bajo esta consideración generalizada de decadencia que se mantiene de forma inamovible en los argumentos históricos de los investigadores europeos durante casi 200 años.

\section{B) Vigencia de la «crisis» en la historiografía española: destrucciones $y$ tesorillos. Las invasiones}

La existencia de una profunda crisis localizada en el s. III, con unas consecuencias poco menos que catastróficas para el posterior desarrollo de la historia de Hispania, ha sido un lugar común en la historiografia española anterior a la década de los 80 . Hasta principios de los 70 esta crisis 
se debía a un factor exógeno al mundo hispanorromano como eran las invasiones de pueblos bárbaros. Este dato se extraía de una información contenida en todas las fuentes literarias disponibles para este periodo, a excepción de la Historia Augusta. Aurelio Victor nos habla de francos, mientras Eutropio, Jerónimo, Orosio y Próspero lo hacen de germanos pero, en ambos casos, la fecha aportada es mas o menos la misma, haciéndola coincidir con el gobierno de Galieno. El único dato que se aporta es el asedio de Tarraco. A pesar de la evidente parquedad de estas noticias, su influencia ha sido decisiva en la historiografía española. El hecho de ser el único testimonio escrito disponible para la mayor parte del s. III le ha concedido un protagonismo trascendental, que supera las posibilidades de lectura que contiene.

Como ya veiamos cuando analizábamos el nacimiento del concepto de crisis aplicado al conjunto del bajo Imperio, pero con sus orígenes cimentados en la Anarquía Militar, era la inestabilidad política y militar la que determinaba, de acuerdo con el desarrollo de una historia positivista y descriptiva, una visión decadentista de todos los elementos constitutivos del Imperio. En el caso de Hispania, la falta de fuentes escritas que la vincularan, de alguna manera, con la inestabilidad política existente en el resto del Imperio y la necesidad de incluirla en un contexto general de crisis llevó a potenciar la acción devastadora de los pueblos germanos.

Por su parte, los restos arqueológicos analizados de este periodo lo han sido siempre de acuerdo con este apriorismo histórico. Motivo por el que, en una especie de círculo vicioso, sus resultados venían siempre a corroborar las devastadoras invasiones. Niveles de destrucción e incendio, tanto en ciudades como en asentamientos rurales, ocultaciones de monetal en forma de «tesorillos", reducción del perímetro urbano y reutilizaciones de material constructivo en la elaboración de las nuevas murallas, surgidas ante el peligro de nuevas incursiones, han sido los argumentos arqueológicos esgrimidos para defender la intensidad de las invasiones y convertirlas en las desencadenantes de una profunda crisis. La falta de material cerámico, que permitiera una datación cronológica más o menos ajustada, ha permitido a los investigadores amoldar los niveles de supuesta destrucción a cronologías precisas para hacerlas encajar en las fechas del relato histórico.

Los tesorillos se han considerado piezas clave en la valoración del paso de las invasiones y sus efectos. Los análisis de A. Blanchet (Blanchet, 1900) y H. Koethe (Koethe, 1950) para la Galia fueron transportados al caso de Hispania con unos resultados menos esclarecedores y válidos. Se quería también, aquí, hacer coincidir la última fecha de acuñación de estas 
ocultaciones con el paso de las invasiones, de esta forma se conseguiría trazar el recorrido de estos pueblos por la Península, completando, de este modo, la parca información de las fuentes escritas. Sin embargo, las fechas y los recorridos no podian encajarse en un único itinerario, lo que provocó la necesidad de considerar dos invasiones sucesivas frente a la única referida en los textos clásicos. Esta idea lanzada por B. Taracena en 1950 (Taracena, 1952) tendrá una buena acogida en la historiografía española siendo aceptada por todos los autores del momento a excepción de A. Balil.

Estamos de acuerdo con J. Arce (Arce, 1978, 265) cuando afirma que esta segunda invasión surge de la necesidad de explicar fenómenos arqueológicos. Pero esta necesidad no tiene su origen, como este autor parece insinuar, en los propios datos arqueológicos, sino en la exigencia de unos esquemas de investigación dependientes exclusivamente de las fuentes escritas. Si los textos hablan de invasión y se consigue asociar esta invasión a unos ítems arqueológicos determinados, de acuerdo a un razonamiento inductivo, siempre que se documenten estos ítems podremos deducir la existencia de este tipo de fenómeno histórico. Con esto no se está hipervalorando la información arqueológica, sino que se está interpretando de acuerdo a un apriorismo histórico que, en este caso, lleva incluso a generar una nueva invasión, no documentada textualmente, para mantener vigente una línea interpretativa.

La crisis del s. III no aparece tratada de forma monográfica para la Península Ibérica hasta la década de los cincuenta, lo que supone un considerable atraso respecto de la investigación europea. De hecho, toda la historia bajoimperial está, hasta estos momentos, elaborada en forma de "Historia Eclesiástica" (Balil ya señalaba este hecho (Balil, 1967, 245), un genero con larga tradición en nuestro país (García Villoslada, 1979, $X I X)$. La falta de una potente burguesía liberal en España y, posteriormente, la marcada orientación católica del Régimen Franquista supone una intensa vinculación entre Iglesia y Cultura que explicaría la falta de estudios sobre el bajo Imperio realizados desde una perspectiva laicista al menos hasta mediados de siglo.

Con anterioridad a los cincuenta contamos con escasos trabajos que refieran algún tipo de mención a este momento de la historia de Hispania. Cronológicamente J. B. Mispoulet en 1910 (Mispoulet, 1910) es el primero en hacer algún tipo de mención al s. III, en un artículo demasiado breve para el amplio tema que aborda, proporcionándonos una información prácticamente anecdótica. Igual sucede con la obra de E. Albertini (Albertini, 1923) publicada en París en 1923, en la que pretende trazar los límites 
administrativos de la provincia hispana, incluyendo las divisiones bajoimperiales. En 1940, R. Thouvenot presenta su trabajo monográfico sobre la Bética (Thouvenot, 1970, reed.) llegando a unas paradójicas reflexiones, si tenemos en cuenta el temprano momento en que éstas se producen. Por primera y última vez en la historiografía española, hasta la década de los 80 , se matiza el concepto de crisis asociado al bajo Imperio y se minimiza la influencia destructiva de los invasores germánicos. Curiosamente, el trabajo de Thouvenot no aparece entre las fuentes consultadas por la historiografía inmediatamente posterior, condenado al olvido prácticamente hasta que se produzca su reedición en 1970.

Más repercusión historiográfica tendrá la monografía dedicada a Ampurias editada en 1934 (Bosch-Gimpera, 1934). La vinculación de la destrucción de esta ciudad a las invasiones germánicas del s. III, a partir del análisis de P. Bosch-Gimpera y su equipo hacen del registro arqueológico, se convertirá en un argumento hartamente usado para ejemplificar tanto la virulencia de las invasiones como la decadencia urbana que provoca la actuación de los bárbaros en la península (Taracena, 1952; Tarradell, 1955 a; Balil, 1957...). Otro estudio arqueológico, el dedicado al denominado «palacio romano» de Clunia realizado por B. Taracena (Taracena, 1946) incide también en sus últimas páginas en la asociación de su nivel de destrucción con el paso de los germanos. El hallazgo de un tesorillo en los niveles de incendio, con una cronología para la moneda más moderna de 284 supone, sin embargo, un problema para esta afirmación ya que las fuentes recogen una invasión en tiempos de Galieno, aproximadamente fechable en 257-258. Este problema cronológico será superado por este autor en la conferencia pronunciada en 1950, al elevar a dos las invasiones. "Las invasiones germánicas en España durante la segunda mitad del s. III d. C." (Taracena, 1952) constituye el primer trabajo monográfico dedicado al $\mathrm{s}$. III en nuestro país. Su importancia historiográfica es excepcional ya que, por un lado, sienta las bases por las que ha de discurrir la investigación histórica y arqueológica hasta al menos mediados de los $70 y$, por otro, es un verdadero revulsivo para el estudio de este periodo. Otro artículo de B.Taracena (Taracena, 1952), uno de J. Sánchez Real (Sánchez Real, 1951) y sendos de M. Tarradell (Tarradell, 1955 a y 1955 b) se publican en los años siguientes ahondando en la línea de investigación ya esbozada y potenciando la imagen de las invasiones como causa última de la crisis que atraviesa la Península Ibérica en la segunda mitad del s. III, crisis que se perpetuará con matices a lo largo de todo el periodo bajo Imperial.

En 1956, cuando se celebre el / Congreso Español de Estudios Clásicos, la "crisis del s. IIl» ya ha adquirido una relevancia propia en la in- 
vestigación española. Esto se traduce en la existencia de una sesión completa, dirigida por A. García y Bellido, dedicada a este tema. M. Tarradell, tras la muerte de Taracena, es el encargado de presentar la ponencia introductoria (Tarradell, 1958) bajo el título "La crisis del s. III en Hispania. Algunos aspectos fundamentales». Estamos ante la asunción definitiva de la historiografía española de la "carencia de estudios globales sobre el periodo imperial frente a la potenciación del periodo republicano debido a la mayor abundancia de fuentes escritas" (Tarradell, 1958, 265). De esta afirmación se deduce, además, el escaso peso que aún tenia la información arqueológica en el proceso de reconstrucción histórica. $M$. Tarradell incide en la sistematización de las invasiones que había planteado en sus dos artículos anteriores, tesorillos y niveles de destrucción arqueológica marcarían el paso de los pueblos bárbaros por la península.

Esta ponencia va acompaña de tres comunicaciones dedicadas también a la "crisis del s. III", una realizada por A. Ramos Folques (Ramos Folques, 1958) y dos por A. Balil (Balil, 1958a, 1958b). El primero de los investigadores presenta una comunicación en la que relaciona, aún con ciertas reservas, el nivel de destrucción localizado en la Alcudia de Elche con el paso de las invasiones germánicas. En otro artículo posterior (Ramos Folques, 1960) vinculará definitivamente ambos hechos, ampliando además su campo de estudio a la provincia de Alicante y asentando el binomio destrucción-invasiones.

A. Balil, que ya había iniciado su estudio de las fortificaciones bajoimperiales (Balil, 1957b), presenta en este congreso dos comunicaciones que preludian su fertilidad posterior. Este investigador, sin apartarse de la teoria de la existencia de una crisis en la segunda mitad del s. III en Hispania, provocada en última instancia por la acción de los pueblos bárbaros, introduce interesantes novedades al tratamiento que en este momento se está dando del tema. Realiza un estudio más riguroso de los documentos arqueológicos, no dejándose llevar por el silogismo expresado por Taracena, y partiendo siempre de la base de la necesidad de contar con un mayor volumen de datos arqueológicos para realizar un análisis ajustado de la realidad. Así, Balil expresa una dura crítica a la investigación que en este momento se está realizando en España: «No creo que sea ya superfluo insistir en la necesidad de un mayor rigor metodológico en la valoración de los resultados de la investigación arqueológica, puesto que casi siempre se procede apriorísticamente" (Balil, 1959, 272). De igual manera, adoptará una actitud crítica con la supuesta segunda invasión, hipótesis invalidada tanto por el silencio de las fuentes escritas como por los escasos restos arqueológicos que en teoria vendrían a corroborarla (Balil, 1959, 280-281). 
Las reflexiones críticas referidas a la metodología y teoría arqueológica son abundantes en la obra de Balil, llegando a realizar verdaderos análisis historiográficos. Su conocimiento de la bibliografía al uso en el resto de Europa le convierte en un precursor de ideas, que debido a la endémica cerrazón española a las corrientes históricas foráneas, tardarán mucho tiempo en incorporarse al análisis histórico en nuestro país. De hecho en 1967 (Balil, 1967) se publica "De Marco Aurelio a Constantino. Una introducción a la España del Bajo Imperio" un largo y denso artículo en el que por primera vez, en la historiografía española, se intenta delimitar el concepto de "crisis" abandonando el cómodo uso de un tópico. Al igual que Vogt (Vogt, 1968), Balil atrasa el inicio de la crisis al s. II presentando numerosos síntomas que nos alertan de un resquebrajamiento, por la propia evolución histórica del Imperio, del sistema ideado por Augusto.

En este sentido, el investigador español se sitúa en la vanguardia del pensamiento histórico europeo, que comienza a realizar un análisis cada vez menos apriorístico y conservador respecto de la historiografía anterior. Balil es un investigador incalificable. Posee un dominio exhaustivo de la historia general de Roma que reivindica como clave, frente a la actitud de la investigación española contemporánea, para entender los acontecimientos del s. III en Hispania. Al mismo tiempo, es capaz de realizar unos análisis arqueológicos que, aún en la actualidad, se utilizan como paradigma de estudio riguroso, tal y como sucede con su exposición sobre las murallas de Barcino (Balil, 1961).

Con el paso del tiempo, sus argumentos van llenándose de matices que implican una visión más compleja de la "crisis" del s. III. Así, el proceso de amurallamiento general, que experimentan muchas ciudades en el último tercio del s. III, se interpreta en 1970 no sólo como efecto de la amenaza exterior, su tesis inicial, sino también debida a una inquietud interna provocada por una crisis social y económica que podía derivar en violencia (Balil, 1970). A pesar de este enriquecimiento paulatino del concepto de "crisis", Balil continua defendiendo la entrada de los pueblos bárbaros como detonante último de las convulsiones políticas, militares y sociales que se viven en Hispania en la segunda mitad del s. III.

La otra gran figura historiográfica de la década de los 60-70 es J. M. Blázquez. La tremenda versatilidad de este investigador, con obras sobre todo el periodo antiguo de la Península Ibérica, lo hace una figura ineludible para la historiografía española no sólo en el estudio del s. III. Su primera incursión en este tema se produce en 1964 con la obra Estructura económica y sociedad de Hispania durante la Anarquía Militar y el Bajo Imperio (Blázquez, 1964). Editada por los Cuadernos de la Cátedra de 
Historia Antigua de España, bajo los auspicios de Viñas y Mey, es el primer trabajo específico acerca de la economía bajoimperial publicado en España.

Con anterioridad tan sólo el citado C. Viñas y Mey (Viñas y Mey, 1959) y G. Lachina (Lachina, 1961) habian dedicado trabajos menores a este respecto. Blázquez articula su estudio por provincias presentando una descripción de la economía y la sociedad bajoimperial sin evolución diacrónica. Al final del estudio, dedica unas páginas al «impacto de las invasiones germánicas del siglo III en la economía" (Blázquez, 1964, 163-182). Ciudades y villas arrasadas, destrucciones generalizadas, desestructuración económica y social, «miseria y ruina en gran parte de la Península y norte de Africa" es el balance que dejan estos pueblos a su paso por Hispania (Blázquez, 1964, 170). Conscientemente, en este trabajo no se pronuncia sobre el número de invasiones, su recorrido o los pueblos que se incluían en ellas, presentando exclusivamente su desastroso impacto sobre una sociedad que hasta estos hechos presentaba símbolos inequívocos de esplendor. Desde el primer momento, como vemos, Blázquez adopta la teoría de una profunda crisis provocada por la llegada de invasores bárbaros.

En 1968 desarrolla estas hipótesis en lo que constituye su última aportación real a la historiografía del s. III (Blázquez, 1968). En lo sustancial, continua la línea de investigación de Taracena y Tarradell, aunque su reflexión se hace extensiva también a la Mauritania Tingitana. Aporta nuevos ejemplos arqueológicos de destrucciones, que se sitúan a lo largo de toda la Península. Así, los niveles de destrucción de Ampurias, Barcino, Caesaraugusta, Liédena, Clunia, Itálica, las ciudades de la costa levantina, Malaga, Baelo y Emerita muestran la intensidad del raid germano y hasta que punto fue destructiva su acción (Blázquez, 1968, 8-14). Los tesorillos, también, son símbolos inequívocos del terror en el que se vio sumida la población y marcan con su presencia el recorrido de las dos invasiones que asolaron Hispania. La fortificación generalizada de ciudades en la segunda mitad del siglo se justifica derivada, de igual modo, de este clima de inseguridad.

Su argumentación no difiere lo mas mínimo, como ya comentábamos, de la expresada con anterioridad por Taracena y Tarradell, sin que podamos encontrar reflexiones novedosas. Se cimienta, además, en unos datos arqueológicos analizados, una vez más, desde el apriorismo histórico y a los que no somete a un análisis riguroso. Blazquez volverá sobre este tema en, al menos, tres ocasiones más (Blázquez, 1973, 1982a, 1982b) pero ni sus planteamientos, ni los datos que maneja, ni sus conclusiones 
finales diferirán de lo ya expresado en su artículo de 1968. A pesar de que las aportaciones de este investigador no suponen un avance real en el conocimiento del s. III, su importancia en la historiografía posterior es evidente. No sólo es un punto de referencia bibliográfica ineludible sino que sus tesis aún serán defendidas por algunos investigadores hasta momentos más o menos recientes (Santos Yanguas, 1986).

Dentro de esta primera etapa de investigación que engloba todos los estudios realizados sobre el $\mathrm{s}$. III hasta aproximadamente principios de la década de los 70 , el concepto de "crisis" es inexcusable y éste se hace extensible a todos los ámbitos de la vida en Hispania. Esta "crisis" tiene un origen siempre militar o político siendo las invasiones las detonadoras de todo el proceso de decadencia.

\section{C) Vigencia de la crisis en la historiografía española: desestructuraciones internas}

A principios de la década de los 70 comienza a percibirse en los estudios sobre la Antigüedad Clásica en España la entrada de nuevos esquemas interpretativos fundamentados en la creación de modelos teóricos. Su vigencia e influencia posterior va ser escasa, no prolongándose, en líneas generales, más allá de la década de los 80 . La exigua implantación de estos modelos en la historiografía española puede explicarse por distintos motivos. En primer lugar, estas líneas de investigación habían empezado a ser superadas en Europa en el mismo momento en que aquí empiezan a dar sus frutos. Se había demostrado la dificultad de reducir a un esquema teórico la complejidad del entramado histórico y comenzaban a ser efectivas las revisiones de los planteamientos metodológicos de estos modelos interpretativos (Hernando, 1992, 21-23). Por otro lado, va a coincidir temporalmente con el inicio del auge de la arqueología en nuestro país. Esto se traduce en una multiplicación de excavaciones que arrojaban unos datos que cada vez iban matizando más la idea de destrucciones generalizadas y que ponian de relevancia la trascendental importancia que el registro arqueológico tiene para la interpretación del s. III, frente a la abrumadora falta de fuentes escritas, base de los estudios de corte teórico.

El primer investigador que llevará a cabo un análisis de corte marxista para el final del mundo antiguo asociado a la decadencia bajoimperial será M. Vigil. En 1967 en su artículo «La Península Ibérica y el fin del mundo antiguo", dentro del libro Las raíces de España (Vigil, 1967), presenta las contradicciones internas en las estructuras sociales y económicas como el 
origen real de la crisis, minimizando la influencia de las invasiones. Los trabajos posteriores de este autor continuarán en esta línea (VIGIL, 1973), afianzando la idea de que la crisis surge de la lucha desarrollada en el s. III entre dos economías contrarias como eran la esclavista y la feudal. El triunfo de esta última supondrá el fin de las estructuras «típicamente» romanas y la implantación de un nuevo modelo económico, político y social debido a la feudalización de las estructuras del Imperio. En este marco la desaparición de la ciudad como célula base del mundo romano es ya un hecho tras el siglo III.

A partir de un análisis marxista se desarrolla también el estudio de J. Fernández Ubiña. Este historiador localiza en el s. III el inicio de la "crisis del estado esclavista", y aunque admite una cierta recuperación económica para el s. IV, la debilidad estructural del sistema lleva irremediablemente a la desintegración del estado romano (Fernández Ubiña, 1982, 24). En su obra La crisis del s. III y el fin del mundo antiguo (Fernández Ubiña, 1984) consolida sus postulados anteriores manejando un mayor volumen de información y llegando a conclusiones más argumentadas. Al mismo tiempo, realiza un completo análisis historiográfico sobre la investigación precedente (Fernández Ubiña, 1984, 91-102) que supone el primer "estado de la cuestión» realizado en España, si excluimos las reflexiones de Balil.

Partiendo de un análisis antropológico, influenciado por la antropología histórica norteamericana, se desarrollan las investigaciones de Ju. B. Tsirkin (Tsirkin, 1987) y Urruela (Urruela, 1981). En ambos casos su influencia en estudios posteriores ha sido prácticamente nula. El peso global en la historiografía española de los modelos de interpretación histórica, ya sean marxistas o a partir de presupuestos antropológicos, ha sido minimo, los motivos bien pueden ser los expuestos arriba.

\section{LA MATIZACIÓN DEL CONCEPTO DE «CRISIS" EN LA HISTORIOGRAFÍA ESPAÑOLA}

Mientras en la década de los 70 la historiografía española seguía defendiendo una visión catastrofista de los acontecimientos del s. III y proyectando hacia el bajo Imperio una decadencia urbana, económica y política, en Europa comienzan a aparecer voces críticas, que en principio matizan el concepto de caducidad vinculado al bajo Imperio para más tarde negarlo. Esta etapa histórica, se convierte así poco a poco, en un periodo con una idiosincrasia propia que no podía seguir siendo contemplada desde los esquemas altoimperiales. Comienza a usarse el termino de "Antigüedad Tardia" que abandona la consideración peyorativa que 
contenía el de «bajo Imperio" y dota a este periodo de una entidad propia, como un momento histórico que posee unas características específicas negando, así, un principio comparativo definitorio.

El debate teórico que queda abierto dentro de la disciplina arqueológica tras la irrupción de la "Nueva Arqueología" supone un revulsivo, ya no sólo para la valoración y cuestionamiento de sus principios metodológicos, sino también de sus principios epistemológicos. A la larga, esto traerá consigo un debate intensivo sobre el propio objeto de estudio y la relación existente entre él y el investigador. Sin poder ni siquiera esbozar aquí el interesante debate teórico desarrollado en las últimas décadas en torno a esta disciplina (Hodder, 1988), es interesante anotar que la implantación de contenidos teoréticos en estos estudios enfocados a la elaboración de una filosofía específica de esta ciencia ha modificado ostensiblemente la apreciación global del proceso cultural e histórico. En el caso que nos ocupa, esto puede traducirse en la valoración de épocas anteriormente consideradas marginales o decadentistas.

También en el ámbito de la Historia Antigua se producen interesantes innovaciones. La lectura crítica de las fuentes comienza a generalizarse provocando interpretaciones novedosas y desterrando mitos que durante largo tiempo habían estado paralizando a la investigación histórica. Los movimientos descolonizadores sirvieron, además, para reivindicar la riqueza cultural de civilizaciones tradicionalmente consideradas inferiores por Occidente que pasan a valorarse, de acuerdo a los principios del "relativismo cultural", desde sus propios esquemas. Probablemente esta modificación en la consideración de "culturas avanzadas" y "culturas menores" tenga mucho que ver con la revalorización de periodos tradicionalmente considerados de decadencia o crisis.

Sea como fuere, en la década de los 70 comienza a cuestionarse esa crisis general que antes era aplicada al s. III y, sin negar la información transmitida por las fuentes escritas de convulsiones políticas y militares, se empieza a acotar el carácter de dicha "crisis". Probablemente sea en la obra de J. Vogt (Vogt, 1968) publicada originalmente en 1965, donde por primera vez aparece matizada la noción de crisis. Si bien el titulo hace referencia a La decadencia de Roma, el subtítulo reza un significativo Metamorfosis de la cultura Antigua, el bajo Imperio aparece, de esta manera, como un interesante momento de cambio cultural. Vogt aún habla de "bajo Imperio", aunque específica que no esto no conlleva para él ningún tipo de consideración peyorativa.

P. Brown en 1971 publica The Wold of Late Antiquity (BROWN, 1989) una obra trascendental en el cambio de enfoque que se estaba operando 
ya respecto al bajo Imperio y a la crisis del s. III. Para este autor existe una continuidad estructural desde el año 200 al año 700 que en ningún caso puede interpretarse como producto de una ruptura dramática con respecto al alto Imperio. Significativo es el uso, ya en este autor, de un nuevo termino para designar los momentos finales del Imperio Romano. A partir de ahora se tenderá a sustituir la designación de «Bajo Imperio" por la de "Antigüedad Tardía". Esta modificación contiene no sólo una importante carga simbólica, al abandonar un término cargado de consideraciones peyorativas, sino que permite además acotar un periodo histórico más coherente. La caida del Imperio Romano fijada, en la historiografía tradicional para la zona occidental, en el mandato de Rómulo Augústulo carece de contenido histórico real. El concepto de Tardía Antigüedad admite una delimitación temporal posterior abierta a los distintos fenómenos que se están produciendo tanto en el unitario bloque oriental como en el fragmentario occidental.

Sin poder revisar aquí la evolución de los estudios sobre el s. III fuera de las fronteras españolas, debemos anotar, sin embargo, algunos de los trabajos que marcan el cambio de concepción que se está produciendo en la segunda mitad de la década de los setenta. En 1978, P.Brown (Brown, 1978) incide con más fuerza en la idea de no poder considerar esta etapa como de decadencia. H. Marrou (Marrou, 1977) realizará una valoración incluso positiva de este periodo al presentar la riqueza de una sociedad que nace de la fusión de dos elementos culturales distintos como son el latino y el germano. Y, por primera vez, se presenta un estudio que cuestiona la debilidad de la ciudad en el s. III a partir del análisis intensivo de un ejemplo concreto. A. Chastagnol (Chastagnol, 1978) realiza un estudio sobre Timgad en el que pone fin al mito de la decadencia urbana, al apreciar en la ciudad africana unas muestras de vitalidad urbana que no se corresponden con los esquemas sombríos anteriores. La arqueología comienza a definirse como una herramienta trascendental para el estudio del s. III y los datos que arroja cada vez matizan más la visión tradicional aportada en primera instancia por las fuentes.

En España, el punto de inflexión historiográfica puede marcarse con cierta seguridad en el trabajo de J. Arce de 1978 (Arce, 1978). Con anterioridad tan sólo F. Rodríguez Neila (Rodríguez Neila, 1972) habia hablado de una cierta continuidad estructural, en lo económico, del bajo Imperio con respecto del periodo precedente, pero aún seguía defendiendo dos invasiones virulentas que habían supuesto el fin de la vida urbana. Arce introduce valoraciones y planteamientos absolutamente novedosos respecto de la historiografía anterior. El título de su artículo "La "crisis" del S. III d. C. en Hispania y las invasiones bárbaras" no rompe a primera vista 
con los postulados básicos de la argumentación tradicional, tan sólo el entrecomillado de crisis nos advierte de una cierta disensión. Esconde, sin embargo, una dura revisión teórica de los presupuestos anteriores, criticando abiertamente la falta de rigor metodológico de Tarradell y Blázquez. De hecho, la propia estructura del artículo coincide con la utilizada por este último autor en uno de sus artículos (Blázquez, 1968) desmontando, con una argumentación aplastante, todos y cada uno de los tópicos acuñados por la historiografía de los años 60 y 70 . Arce no realiza en este estudio una ruptura radical, entendiendo esto como una exposición de nuevos puntos de vista, sino que se dedica concienzudamente a rebatir las posturas anteriores. Plantea la necesidad de conceptualizar el termino de "crisis" para, en todo caso, dotarlo luego de un contenido histórico. Realiza una lectura crítica de las fuentes para demostrar su falta de inocencia en la exposición de los hechos. Los textos nos hablan desde una posición prosenatorial y claramente contraría a Galieno. La corroboración arqueológica, que sustentaba a través de destrucciones y tesorillos la virulencia de las invasiones, es también analizada desde el rigor y los últimos datos arqueológicos disponibles. El amurallamiento de las ciudades debe contemplarse como un símbolo de ennoblecimiento y no de decadencia, siguiendo a R. Rebuffat (Rebuffat, 1974). Como conclusión, afirma que la «crisis», si es que existió, no puede fundamentarse en la acción de los pueblos bárbaros que irrumpieron en el s. III en la Península. El mismo reconoce al final de su artículo (Arce, 1978, 269) que su objetivo no era intentar delimitar el verdadero alcance de la crisis, sino desmontar una argumentación que llevaba ya demasiado tiempo sosteniéndose sin argumentos convincentes. Será en trabajos posteriores cuando Arce (Arce, $1982,1987,1988$ ) dé el siguiente paso y construya una imagen del bajo Imperio alejada ya de dramatismos y decadencias. La ciudad no muere en el s. III sino que asiste, como el resto de los aspectos de la cultura romana, a una redefinición que le permite amoldarse a los nuevos tiempos.

Podemos localizar un segundo hito historiográfico en el I Symposium numismático de Barcelona, celebrado en 1979. En una de las sesiones de este congreso, "La circulación monetaria en el Mediterráneo occidental", se abordan los problemas derivados de la utilización de las fechas de acuñación en la datación del registro arqueológico y la necesidad de valorar otra serie de variables intrínsecamente unidas a la propia naturaleza de la rnoneda (Campo, 1979; Campo y Granados, 1979; Bost et alii,1979; Gurt, 1979a y 1979b; Hiernard, 1979a y 1979b; Manera y Granados, 1979). Las conclusiones allí expuestas sentarán las bases para el desmoronamiento definitivo de la vinculación tesorillo-invasión, al plantear una renovación metodológica que implica un enriquecimiento del análisis numismático. 
Fruto directo de las disertaciones expuestas en este congreso es el artículo de M. Campo y J. M. Gurt publicado en 1980 (Campo y Gurt, 1980). En él se critica la deliberada y continua adaptación de los resultados arqueológicos para hacerlos coincidir con las invasiones germanas (Campo y Gurt, 1980, 129) y se plantea la necesidad de diferenciar "deposito" de "tesorillo", ya que uno u otro fenómeno arqueológico esconde realidades históricas bien diferentes. Esta diferenciación sólo puede hacerse con un conocimiento ajustado del monetal en circulación en un determinado momento. Tan sólo las ocultaciones pueden estar mostrando el avance de un peligro o de una situación de inseguridad, lo que no puede constatarse para el caso de Hispania.

La renovación de los estudios numismáticos que se produce en España en la década de los 80 , con un retraso evidente, una vez más, respecto del resto de Europa, invalidará la teoría de una profunda crisis económica originada en el s. III y provocada por la entrada de los pueblos bárbaros. En esta década se publicarán distintos trabajos en este sentido (Sagredo, 1981, 1985; González Prats y Abascal, 1987) dispuestos a anular definitivamente uno de los pilares en los que se fundamentaba la investigación precedente para sostener su hipótesis decadentista.

A partir de este punto, uno a uno irán cayendo los sustentos de la noción de crisis aplicada al s. III. Anulada la posibilidad de trazar el recorrido de estos pueblos a través de los hallazgos numismáticos, realizada una lectura crítica de las fuentes que situara en su justa medida la afirmación del caos, tan sólo quedaba ya la revisión de la imagen de destrucción y decadencia urbana que la arqueologia poco a poco va ir realizando en esta década de los ochenta. La multiplicación de las excavaciones urbanas, arropadas por la Ley de Patrimonio de 1985, ha permitido contar con un importante volumen de datos que cuestionaban el simplismo reduccionista de la afirmación de "muerte urbana". En los 80 , en resumen, se sientan las bases para una concepción del s. III como preludio de una nueva realidad, no definida ya a partir de la comparación peyorativa con el periodo precedente. En los noventa aparecerán trabajos, no dispuestos a desmontar las viejas visiones (esto se ha realizado ya en la década precedente) sino a aportar argumentos novedosos y nuevas explicaciones para los fenómenos de la "Tardía Antigüedad".

Como ya deciamos, en vez de hablar de crisis urbana comienza a imponerse la tesis de una modificación del paisaje de la ciudad respecto de los modelo altoimperiales. Junto con la publicación de los niveles tardios de distintas ciudades, aparecen trabajos de síntesis que pretenden trazar la situación de la ciudad en los siglos bajoimperiales. Cabe destacar el 
artículo de J. M. Gurt, G. Ripoll y C. Godoy (Gurt et alii, 1994) en el que se hace hincapié en la necesidad de aislar los nuevos fenómenos de ocupación vinculados a la cristianización del territorio. El s. III aparece como puente entre dos realidades culturales distintas en las que priman las continuidades frente a las rupturas. En la misma línea se producen las reflexiones de A. Fuentes Domínguez (Fuentes, 1997) que realiza un intento de conceptualización de la nueva realidad urbana al introducir el concepto de "isostasia" (Fuentes, 1997, 479) para definir la reordenación del ámbito de influencia de las distintas ciudades hispanas de acuerdo con la nueva situación política, religiosa y económica.

Con anterioridad C. Fernández Ochoa y A. Morillo Cerdan (Fernández Ochoa y Morillo, 1991, 1992) habian publicado una completa revisión de las murallas bajoimperiales descartando el tópico generalizado de la reducción del perímetro urbano y negando la validez de la vinculación entre reutilización de material e invasión, sin otros datos arqueológicos que la avalen (Fernández Ochoa y Morillo, 1992, 347). Potencian además la idea de que las fortificaciones tardías de la Península lbérica, con excepción tal vez de las del noroeste peninsular, no se deben a un plan conjunto y coordinado ante una amenaza externa concreta, sino que tienen un origen independiente en cada una de las ciudades debida a sus propias necesidades, no necesariamente defensivas (Fernández Ochoa y Morillo, 1992, 348).

El siglo III en la historiografía actual no se define ya como un siglo de crisis frente al esplendor del s. II; se define ahora como periodo de transito a la nueva realidad cultural característica del mundo tardorromano. Es decir, ha pasado de formularse negativamente frente a un periodo anterior, a hacerlo como un periodo de transición en el que cabe encontrar un buen número de continuidades pero también el germen de los nuevos fenómenos historico-arquelógicos característicos de los s. IV y $\mathrm{V}$. Volvemos a enfrentarnos con un periodo que aún no puede evaluarse desde sus propios acontecimientos y realidades culturales. La falta de fuentes escritas que condicionó la investigación anterior a los 80 no ha sido paliada con el conocimiento arqueológico que aún es fragmentario en numerosos aspectos. De hecho, superada la polémica de las invasiones, el s. III se ha visto desplazado en el interés de los investigadores, con evidentes excepciones, en la historiografía de la última década. El análisis y conceptualización de la nueva realidad tardía ha centrado el interés de los investigadores dedicados al bajo Imperio, mientras el s. III continua anclado en su indefinición temporal, convertido en apéndice del alto Imperio y en preludio del S. IV.

De este modo, la publicación de una obra monográfica sobe el s. III era una necesidad ineludible para la investigación actual. Eran numerosos 
los datos arqueológicos dispersos en una fragmentada bibliografía y se hacia necesario ya, como paso previo al conocimiento de este siglo, un estudio crítico de la historiografía precedente una vez que se había conseguido desterrar la inamovible imagen de crisis acuñada en la década de los cincuenta. A. Cepas aborda esta tarea en su obra Crisis y continuidad en la Hispania del siglo III (Cepas, 1997). En ella realiza una exhaustiva búsqueda bibliográfica con el objetivo de compilar toda la información dispersa relativa al s. III. Especialmente interesante nos resulta, desde el enfoque urbano inicial de nuestro trabajo, el análisis realizado sobre un amplio catalogo de ciudades, intentando detectar su evolución a lo largo del s. III. Sin embargo, las conclusiones son necesariamente tan vagas como la falta de información disponible. El estudio de Cepas presenta en conjunto toda la información disponible para reconstruir el s. III en la Península lbérica pero, desgraciadamente, la falta de datos es todavía la tónica dominante.

\section{BIBLIOGRAFIA}

Albertini, E. (1923): Les divisions administratives de l'Espagne Romaine. Paris.

ARCE, J. (1978): "La crisis del s. III d. C. en Hispania y las invasiones barbaras", Hispania Antiqua, vol. 8, págs. 257-269.

ARCE, J. (1986): El último siglo de la España Romana: 284-409, Madrid.

ARCE, J. (1987): "La crisis y el Bajo Imperio». Historia General de España y América, vol. 2, págs. 285-291.

ARCE, J. (1988): España entre el mundo antiguo y el mundo medieval. Madrid.

ARCE, J. (1993): “La transformación de Hispania en época tardorromana: paisaje urbano, paisaje rural». III Congreso de estudios medievales. De la Antigüedad al Medievo (siglos IVVIII). Ávila.

BALIL, A. (1957a): "Las invasiones germánicas en Hispania durante la segunda mitad del s, III d. C.". Cuadernos de Trabajo de la Escuela Española de Historia y Arqueologia de Roma, vol. 9, págs. 97-143. También en AHAM, 1957-58 (1959), págs. 49-91.

BALIL, A. (1957b): "La cronologia de las fortificaciones de Barcino en el Bajo Imperio". IV CAN. Zaragoza.

BALIL, A. (1958a): "Los trabajos de fortificación en las provincias del Occidente romano después de la crisis del siglo in y su significación politica, militar y social", Actas del I Congr. Esp. De Est. Clas, Madrid, págs. 281 y ss.

BALIL, A. (1958b): «Sobre la fortificación del Bajo Imperio en Hispania: las puertas de la muralla de Barcino". Actas I Congreso Español de Estudios Clásicos. Madrid.

BALIL, A. (1959): "Hispania en los años 260 a 300 d. C.». Emérita, vol. 27, fasc. 2, págs. 269-295.

BALIL, A. (1959b): "Las invasiones germánicas en Hispania durante la segunda mitad del s. II d. C.". Anales de Historia Antigua y Medieval, págs. 49-91.

BALIL, A. (1960): "La defensa de Hispania en el Bajo Imperio", ZEPHYRUS XI, págs. 179 y sS.

BALIL, A. (1961): Las murallas romanas de Barcelona. Madrid.

BALIL, A. (1963): "Las fortificaciones del Bajo Imperio en las provincias romanas de Hispania". Celticum XIII.

BalIL, A. (1967a): "De Marco Aurelio a Constantino. Una introducción a la España del Bajo Imperio". Hispania, 27, págs. 245-341.

BALIL, A. (1967b): "La España del Bajo Imperio: problemas y perspectivas de estudio ante una nueva etapa de investigación». III Congreso Español de Estudios Clásicos, 177-207.

BALIL, A. (1970): "La defensa de Hispania en el Bajo Imperio. Amenaza exterior e inquietud interna", Legio VII Gemina, León, págs. 601 y ss. 
BARBERo, A. y Vigil, M. (1974): Sobre los origenes sociales de la Reconquista. Barcelona.

BLANCHET, A. (1900): Les trésors de monnaies romaines et les invasions germaniques en la Gaule. París.

BLÁZQUEZ, J.M. ${ }^{a}$ (1964): Estructura económica y sociedad de Hispania durante la Anarquía Militar y el Bajo Imperio. Madrid.

BLÁZQUEZ, J.M.a (1968): "La crisis del s. IIl en Hispania y Mauritania Tingitana», Hispania, vol. 28 , págs. 537 y ss.

BLAZQUEZ, J.M. ${ }^{a}$ (1973): "El Imperio y las Invasiones desde la crisis del s. III al año 500 ", Historia económica y social de España. Vol. I: La Antigüedad, págs. 331-340.

BLAZQUEZ, J. M. ${ }^{a}$ (1978): "Conflicto y cambio en Hispania durante el S. IV". Transformations et conflits an IV siècle a.p. J.C.. Bonn. 53 y ss.

BLAZQUEZ, J.M. ${ }^{a}$ (1982): "La crisis del s. Ill y la Hispania del Bajo Imperio". Historia de España, Menéndez Pidal. Tomo 2: España Romana, vol. 1, págs. 247- 288.

BOSCH-GIMPERA, P. et alii (1934): Emporiom. Barcelona.

BOST, J.P.; CAMPO, M. y GURT, J. M. (1979): "La circulación monetaria en Hispania durante el periodo romano-imperial: Problemática y conclusiones generales". S. N. B., II, 174-202.

BRown, P. (1971): El mundo de la Antigüedad Tardia (Trad. 1989). Madrid.

BROWN, P. (1978): The making of Late Antiquity. Harvard.

CAMPO, M. (1979): “Circulación monetaria en Menorca». SNB, I, 97-110.

CAMPO, M. y Granados, O. (1979): "Aproximación a la circulación monetaria en Colonia Barcino". SNB, I, 57-69.

CAMPO, M. y GuRT, J.M. (1980): “El problema de la crisis del s. III: su reflejo en los hallazgos monetarios realizados en la costa catalana y las Baleares". Numisma, vol. $30, \mathrm{n} .^{\circ}$ 165-67, págs. 129-140.

Cepas Palanca, A. (1997): Crisis y continuidad en la Hispania del siglo III. Madrid.

Chastagnol, A. (1978): L Album municipale de Timgad. Antiquitas R3.

COllingwoOd, R.G. (1952): Idea de la Historia. Méjico.

FERnÁndez OCHOA, C. y MORILlo CERDÁN, A. (1991): “Fortificaciones urbanas de época bajo imperial en Hispania. Una aproximación crítica (primera parte)". CUPAUAM, 18.

FERNÁNDEZ OCHOA, C. y MORILLO CERDÁN, A. (1992): “Fortificaciones urbanas de época bajo imperial en Hispania. Una aproximación crítica (segunda parte)». CUPAUAM, 19.

FERNANDEZ OCHOA, C. (1997): La muralla romana de Gijón (Asturias). Gijón.

FERNANDEZ OCHOA, C. (1998): "La ciudad en la Antigüedad Tardía en la cornisa cantábrica". Complutum y las ciudades hispanas en la Antigüedad Tardía, POLIS, págs. 70-83.

FERNÁNDEZ UBIÑA, J. (1981): La crisis del s. III en la Bética. Granada.

FERNÁNDEZ UBIÑA, J. (1982): La crisis del s. I'l y el fin del mundo antiguo. Madrid.

FUENTES DOMINGUEZ, A. (1996): “La romanidad tardia en los territorios septentrionales de la península ibérica". Los finisterres atlánticos en la antigüedad. Gijón.

FUENTES DOMINGUEZ, A. (1997): “Los ejércitos y otros sintomas de la descomposición social. Fin del Imperio Romano". La guerra en la antigüedad. Una aproximación al origen de los ejércitos en Hispania. Madrid.

FUENTES DOMINGUEZ, A. (1997): «Aproximación a la ciudad hispana de los siglos IV y $\vee$ d. C.». Cong. Int. La Hispania de Teodosio. Vol.2. 477-496.

GaRcia VILLOSLADA, R. et alii (1979): Historia de la Iglesia en España. Tomo I. España romana $y$ visigoda (s. I-VIII). Madrid.

GonzÁLEZ PRATS, A. y ABASCAL, J. M. (1987): “La ocultación monetaria de la D’Eula, Crevillente (Alicante) y su significación para el estudio de las invasiones del s. II!". Lucemtum 6, 183190.

GURT, J. M. (1978): "Hallazgo de un tesorillo del siglo III en la villa romana de Torre Llauder (Mataró)". Gaceta numismática, 44, 81-89.

GURT, J. M. (1979 a): "Circulación monetaria en época imperial en la costa catalana entre los municipios de lluro y Baetulo". SNB I, 71-81.

GURT, J. M. (1979 b): "La circulación monetaria en Rhode (Rosas) durante el Imperio Romano a través de dos colecciones particulares". SNB I, 39-44.

HABERMAS, J. (1968): Ciencia y técnica como «ideología". Madrid.

HERNANDO, A. (1992): "Enfoques teóricos en arqueología». SPAL 1, 11-35.

HIERNARD, J. (1978): "Monedas del s. III en el Museo Arqueológico Provincial de Tarragona". Acta Numismática, VIII, 97-133. 
HIERNARD, J. (1979 a): “Tarragona. Monedas del siglo ill descubiertas en las excavaciones antiguas». SNB, I, 83-89.

HiERNARD, J. (1979 b): “Tarragone: La circulation monétaire au llle siècle apr. J.-C.». SNB II, 49-51.

HODDER, I. (1988): Interpretación en arqueologia. Corrientes actuales. Barcelona.

JUAN TOVAR, L.C. (1997): «Las industrias cerámicas hispanas en el Bajo Imperio. Hacia una sistematización de la T.S.H.T.". Cong. Int. La Hispania de Teodosio, vol. 2, 543-568.

KOETHE, H. (1950): "Zur Geschichte Galliens im drittem viertel des 3.Jahrhunderts". Ver.Röm. Germ.Komm. 32, 1942, 199-224.

LACHINA, G. (1961): “La estructura económica de Hispania en el Bajo Imperio», ZEPHYRUS, XII, págs. 55 y ss.

López Rodriguez, J.R. (1985): Terra Sigillata Hispánica Tardia decorada a molde de la Península lbérica. Salamanca.

MANERA, E. y GRANADOS, O. (1979): “Aproximación a la circulación monetaria en la ciudad romana de Pollentia (Alcudia, Maliorca) hasta el 294 d. de C.". SNB, II, 40-45.

MARROU, H.I. (1977): Décadence Romaine au Antiquité Tardive?. Paris.

MAYET, F. (1984): Les céramiques sigillées hispaniques. Paris.

MAZZARINO, S. (1979, trad. frances): La fin du monde antique. Avatars d un thème historiographique. Paris.

MEZQuiRIZ, M.A. (1961): Terra Sigillata Hispánica. Valencia.

MispoulET, J. B. (1910): “Transformations de l'Espagne durant les trois premiers siècles de l'Empire Romain". Rev. de Philologie, 34, 301 y ss.

OLMOS, R. (1997): "La reflexión historiográfica en España: ¿Una moda o un requerimiento cientifico? ". La cristalización del pasado: génesis y desarrollo del marco institucional de la arqueologia en España. Málaga. 19-28.

ORFILA, M. (1993): "Terra Sigillata hispánica tardia meridional». AespA 66, 125-147.

Paz Peralta, J. A. (1991): Cerámica de mesa romana de los siglos $/ I$ al iv en la provincia de Zaragoza. Zaragoza.

PONSICH, M. (1974): Implantation rurale antique sur le Bas-Guadalquivir. Madrid.

RAMOS FERNÁNDEZ, R. (1964-65): "Las invasiones de los francos en España", Anales de la Universidad de Murcia, XXIII, $\mathrm{n} .^{\circ} 3-4$.

RAMOS Folques, A. (1958): "Las invasiones germánicas en la Alcudia (Elche)", / Cong. Esp. De Estudios Clásicos, Madrid, págs. 275-279.

RAMOS Foloues, A. (1960): "Las invasiones germánicas en la provincia de Alicante", Inst. est. Alicantinos, págs. 9-53.

REBUFFAT, R. (1974): “Enceintes urbaines et insécuritè en Mauritanie Tingitane». MEFR, 501-522.

Rodriguez NEILA, F. (1972): "Aspectos del s. Ill en Hispania", Hispania Antiqua, vol. 2, págs. 179-201.

SAGREDO, L. (1981): "Las invasiones del s. III en Hispania a la luz de los tesorillos monetales", Hispania Antiqua, vol. 11-12, págs. 89-104.

SAGREDO, L. (1983): Circulación e inflación monetaria en la Hispania romana del s. II d. C. Valladolid.

SAGREDO, L. (1985): "Sobre la supuesta invasión del siglo lll d. C. en la Meseta Norte: Palencia", I Coloquio de Historia de Palencia.

SANCHEZ REAL, J. (1951): "La invasión germánica del 259 en Tarragona». Bol. Arq. Tarraconense, LI, fasc. 35-36, págs. 129-131.

SANCHEZ REAL, J. (1957): "Las invasiones germánicas". Bol. Arq., fac. 57-60, págs. 612 y ss.

SANTOS YANGUAS, N. (1986): "Las invasiones germanas del s. Ill en Hispania. Estado de la cuestión", Memorias de H." Antigua, vol. 7, págs. 151-175.

SOTOMAYOR, M. et alii (1979): Historia de la Iglesia en España. Madrid.

TARACENA, B. (1946): «El Palacio Romano de Clunia", AespA, págs. 29-60.

TARACENA, B. (1949): "Las fortificaciones y la población de la España romana», IV Congreso arqueológico del Sudeste español. Cartagena.

TARACENA, B. (1952): "Las invasiones germánicas en España durante la segunda mitad del s. III d. C.". Actas / Congreso Español de Estudios Pirenaicos (S. Sebastian, 1950), vol. 6, secc. 5. págs. 37-45.

TARACENA, B. (1957): «Problemas cronológicos de las invasiones germánicas del siglo $\mathrm{H}$ d. C.", CAN, 4, págs. 227 y ss. 
TARRADELL, M. (1955a): “Sobre las invasiones germánicas del s. III d.C. en la Península Ibérica». Estudios Clásicos, vol. 3, n.15, págs. 95-110.

TARRADELL, M. (1955b): “Problemas cronológicos de las invasiones germánicas del s. III d. C.". CNA IV (Burgos, 1955), págs. 231-239.

TARRADELL, M. (1958): “La crisis del s. III d. C. en Hispania: algunos aspectos fundamentales». 1 Congreso Español de Estudios Clásicos (Madrid, 1956), págs. 263-275.

TARRADELL, M. (1968): "Población y propiedad en el este peninsular durante el Bajo Imperio", III CEEC, 1966 (1968), II, págs. 164 y ss.

TEJA, R. (1974): Organización económica y social de Capadocia en el s. VI, según los Padres Capadocios. Salamanca.

TEJA, R. (1976): «Sobre la actitud de la población urbana en Occidente ante las invasiones barbaras", HAnt VI, págs. 7 y ss.

TEJA, R. (1977): “Honestiores y humiliores en el Bajo Imperio: la configuración de clases de una división juridica". Memorias de Historia Antigua, I, 115 y ss.

THOUVENOT, R. (1940): Essai sur la province romaine de Bétique. París.

TRIGgER, B.C. (1992): Historia del pensamiento arqueológico. Barcelona.

TSIRKIN, Ju. B. (1987): "The crisis of antique society in Spain in the third century", Gerion, vol. 5 , págs. $253-270$.

TUSET, F. y BUXEDA, J. (1995): "La cerámica terra sigillata hispánica avanzada (TSHA) de Clunia: segunada mitad s. II-s. Ill de. C.".l Cong. Arq. Penínsular, 5, 355-367.

URRUELA, J. (1981): Romanidad e indigenismo en el N. peninsular a finales del Alto Imperio. Un punto de vista crítico. Madrid.

VIGIL, M. (1963): «Romanización y permanencia de estructuras sociales en la España septentrional», BRAH, 152, págs. 225 y ss.

VIGIL, M. (1967): "La Península Ibérica y el fin del mundo antiguo», Las raices de España, págs. 283 y ss. Madrid.

VIGIL, M. (1973): Edad Antigua. Madrid.

VIÑAS, C. y MEY, C. (1959): "Apuntes sobre historia social y económica de España", Arbor, $157-8$, págs. 33 y ss.

VOGT, J. (1968): La decadencia de Roma. Metamorfosis de la cultura antigua. Madrid.

VV. AA. (1993): Ciudad y comunidad cívica en Hispania (siglos II y $1 /$ d.C.), Casa de Velázquez, Madrid.

VV. AA. (1997): Actas del Congreso Internacional La Hispania de Teodosio, Segovia. 